\title{
A Goal Programming Methodology for Multiobjective Optimization of Distributed Energy Hubs Operation
}

\author{
M. La Scala ${ }^{\mathrm{a}}$, A. Vaccaro ${ }^{\mathrm{b} *}$, A. F. Zobaa ${ }^{\mathrm{c}}$ \\ ${ }^{a}$ Department of Electrical Engineering, Politecnico di Bari, Bari 70125, Italy \\ ${ }^{\mathrm{b}}$ University of Sannio, Department of engineering, Piazza Roma 21, 82110, Benevento, Italy \\ ${ }^{c}$ Brunel Institute of Power Systems, School of Engineering and Design, Brunel University, Uxbridge, UB8 3PH, \\ Middlesex, United Kingdom \\ *corresponding author: vaccaro@unisannio.it
}

\begin{abstract}
This paper addresses the problem of optimal energy flow management in multicarrier energy networks in the presence of interconnected energy hubs. The overall problem is here formalized by a nonlinear constrained multiobjective optimization problem and solved by a goal attainment based methodology. The application of this solution approach allows the analyst to identify the optimal operation state of the distributed energy hubs which ensures an effective and reliable operation of the multicarrier energy network in spite of large variations of load demands and energy prices. Simulation results obtained on the 30 bus IEEE test network are presented and discussed in order to demonstrate the significance and the validity of the proposed method.
\end{abstract}

Keywords: Energy Hubs, Multicarrier Energy Networks, Optimal Energy Flow Analysis

\section{Introduction}

The energy grids are, in general, amongst the most reliable systems worldwide. These large interconnected infrastructures, however, are subject to a host of challenges as far as aging assets, generation and transmission expansion to meet growing energy demand, distributed resources and reliability coordination are concerned. In this complex scenario, the large scale deployment of the Energy Hub paradigm could play a strategic role in supporting the evolution of conventional energy grids toward active, flexible and self healing networks composed by distributed, cooperative and interactive resources.

From a conceptual point of view, the Energy Hub is a unit where multiple energy carriers can be converted and conditioned. More specifically, they process multiple energy carriers at their input ports and provide certain required energy services (i.e. electricity, heating) at the output ports [1-3]. Consequently, energy hubs could be considered as flexible interfaces between different energy infrastructures (i.e. electrical networks, natural gas distribution systems) and/or energy users (i.e. producers, consumers). Within this interface energy is converted and conditioned by using a wide spectrum of technologies as far as combined heat and power technology, power-electronic devices, and heat exchangers are concerned. These converters allow energy hubs to combine and couple different energy carriers by redundant paths inducing a certain degree of freedom in its supply with several advantages compared to conventional, decoupled energy supply. Many industrial facilities can be modelled according to the energy hub paradigm. They include industrial plants, larger buildings, rural and urban districts, microgrids and isolated energy systems [4,5].

Energy hubs, if properly coordinated and managed, could increase the efficiency of multicarrier energy systems by: (i) allowing the large scale penetration of small-scale distributed generation systems; (ii) supporting the integration of renewable energy sources; (iii) reducing system losses and green house gas emissions; (iv) increasing the reliability of the energy supply to the customers [6,7]. 
As a consequence, a significant growth in the number of Energy Hubs connected to the existing energy distribution systems is expected in the near future.

From this perspective, a crucial issue is how to increase the Energy Hubs efficiency by properly coordinating their operation. In particular, due to the degrees of freedom established by the redundant connections, various energy vectors and different combinations of them can be used to meet the Energy Hub load requirements. This flexibility can be properly exploited to optimize the Energy Hub supply [1,4] since different inputs can be characterized by different costs, availability, and other technical and/or economical criteria.

Many classes of solution algorithms aimed at addressing this problem have been proposed in literature [1-4]. They include linear algorithms, which are based on the linearization of both the objective function and the problem constraints, and nonlinear programming techniques, which deal with problems involving nonlinear objective and/or constraint functions

These solution methods represent an useful tool only from an user perspective, since they allow the analyst to effectively optimize the operation of a single energy hub without considering its impact on the multicarrier energy network operation.

Consequently the research for alternative techniques aimed at optimizing the operation of interconnected and distributed energy hubs by ensuring an effective and reliable operation of the multicarrier energy network is still an open problem and requires further investigations. We refer to this problem as the optimal energy flow problem.

Following this direction, in this paper we propose a solution based on the theory of multiobjective goal attainment optimization. In details we show as the optimal asset of the energy hubs network which (i) meets the loads, (ii) minimizes the energy costs and (iii) assures a robust and reliable operation of the multicarrier energy network can be formalized by a nonlinear constrained multiobjective optimization problem. Since these design objectives conflict with each other, the solution of such the optimal energy flow problem hasn't got a unique solution and a suitable trade off between the objectives should be identified

To address this problem, one of the most common solution approach is based on the weighted global criterion method in which all objective functions are combined to form a single utility function. The main limitation inside these techniques in solving the optimal energy flow problem is that the weighting coefficients of the objective function do not necessarily allow trade-offs between the objectives to be expressed especially when the objectives are competing and the number of objectives increases. Further on the weighting strategies suffer of the so-called convexity problem that, in some cases, does not allow the analyst to explore the whole solutions space. To fix this issue a solution paradigm based on the multiobjective goal attainment methodology is here proposed. The insight principle is to solve the optimal energy flow problem by firstly minimizing each objective function. This asks for the solution of a proper number of constrained scalar optimization problems characterized by different objective functions and the same set of equality and inequality constraints. The obtained solutions (also known as utopia points) are then processed by a goal attainment based programming technique aimed at identifying the final trade off solution.

Simulation results obtained on the 30 bus IEEE test network will be presented and discussed in order to prove the effectiveness of the proposed methodology in the task of solving the optimal energy flow problem in spite of large variations of load demands and energy prices. 


\section{Multicarrier Energy Network Modelling}

\subsection{Electrical power network}

Electrical power system modeling asks for the calculation of the steady-state voltage phasor angle and magnitude for each network bus for a given set of parameters (i.e. load demand and real power generation). Based on this information, all the variables characterizing the actual power system operation point (i.e. the real and reactive power flows on each branch and the power losses) can be computed.

In details, the input (output) variables of the electrical power system model are typically: the real and reactive power (voltage magnitude and angle) at each load bus; the real power generated and the voltage magnitude (reactive power generated and voltage angle) at each generation bus; and the voltage magnitude and angle (the real and reactive power generated) at the slack bus.

The equations used to solve this problem are the real power balance equations at the generation and load buses, and the reactive power balance at the load buses. These equations (also known as power flow equations) can be written as:

$$
\begin{aligned}
& P_{i}^{S P}=V_{i} \sum_{j=1}^{N} V_{j} Y_{i j} \cos \left(\delta_{i}-\delta_{j}-\theta_{i j}\right) \quad i \in n P \\
& Q_{j}^{S P}=V_{j} \sum_{k=1}^{N} V_{k} Y_{j k} \sin \left(\delta_{j}-\delta_{k}-\theta_{j k}\right) \quad j \in n Q
\end{aligned}
$$

where:

- $\quad N$ is the total bus number;

- $\quad n Q$ is the list of the buses in which the reactive power is specified;

- $\quad n P$ is the list of the buses in which the active power is specified;

- $\quad P_{i}^{S P}$ and $Q_{j}^{S P}$ are the real and reactive power injections specified at $i$-th and $j$-th bus;

- $\vec{V}=V_{i} \angle \delta_{i}$ is the $i$-th bus voltage (in polar coordinates);

- $\quad \vec{Y}_{i j}=Y_{i j} \angle \theta_{j i}$ is the $i j$-th element of the bus admittance matrix.

Due to the nonlinear nature of the power flow equations, numerical methods are employed to obtain a solution that is within an acceptable tolerance [8].

\subsection{Natural gas network}

The natural gas network is mainly composed by a system of pipelines with a set of production fields, processing plants, transportations and markets nodes [9].

In our study we assumed that each production node is equipped by a compressor which allows the gas to be injected into the network at a pressure level that can be fixed within certain limits, depending by the compressor and the physical pipeline proprieties.

The natural gas is delivered to the energy hubs by one or more transportation pipelines. For the sake of simplicity we assumed that the gas pressure cannot be increased in the transportation nodes, i.e., there are no compressors available in these nodes.

According to these hypothesis, we can model the flow $q$ in each pipeline in function of the inlet $\left(p_{\text {in }}\right)$ and outlet $\left(p_{\text {out }}\right)$ pressure by the nonlinear Weymouth equation: 


$$
q=k \sqrt{p_{\text {in }}^{2}-p_{\text {out }}^{2}}(2)
$$

Where $k$ is a constant factor whose value depends by the physical properties of the pipeline [10].

By analysing equation (2) it is worth observing that opposing to electricity networks, where no active electrical power is needed to support the buses voltage, the generation of pressure via compressors needs power. In this context, we assumed that the compressors are driven by gas turbines and the corresponding power consumptions are considered as additional power flowing into the pipeline section. According to this hypothesis, the power demand of the compressor installed on the pipeline connecting the nodes $m$ and $n$ can be modelled as [2]:

$$
q_{\text {com }}=k_{\text {com }} q_{m n}\left(p_{m}-p_{k}\right)(3)
$$

where $k_{\text {com }}$ is a constant characterizing the compressor unit while $p_{k}$ and $p_{m}$ denote the suction and discharge pressures respectively. More details on this modelling approach can be found in [1,2].

\subsection{Energy $\mathrm{Hub}$}

The Energy Hub is here modelled by a multi-inputs and multi outputs energy flow converter [4]. In details, for each input variable $E_{\alpha}$ we consider $n$ output $E_{\alpha}^{1}, \ldots, E_{\alpha}^{n}$ variables with:

$$
E_{\alpha}=\sum_{i=1}^{n} E_{\alpha}^{i}(4)
$$

Moreover we denote as $\eta_{\alpha, \beta}^{j}$ the $j$-th converter efficiency characterising the conversion between the input carrier $\alpha$ to the output carrier $\beta$. The input powers to each converter are constrained by minimum and maximum capacity limits:

$$
\mathbf{P}_{\min } \leq \mathbf{P} \leq \mathbf{P}_{\max }(5)
$$

where inequalities are meant component-wise.

Summarizing in a column vector all the variables denoting the input energy flow: $\mathbf{E}=\left[\mathbf{E}_{1}, . ., \mathbf{E}_{\alpha}, . ., \mathbf{E}_{\mathbf{N}}\right]$ where $\mathbf{E}_{\alpha}=\left[E_{\alpha}^{1}, . ., E_{\alpha}^{C}\right]$ and the output energy flow in a column vector $\mathbf{L}=\left[L_{1}, . ., L_{M}\right]$, the resulting formulation for the multi-input multi-output converter is:

$$
\left[\begin{array}{l}
L_{1} \\
. . \\
L_{M}
\end{array}\right]=\boldsymbol{\theta}\left[\begin{array}{l}
\mathbf{E}_{1} \\
. . \\
\mathbf{E}_{\mathbf{N}}
\end{array}\right](6)
$$

where matrix $\boldsymbol{\theta}$ is called the converter coupling matrix whose elements could be zeros, efficiencies or product of efficiencies ${ }^{1}$. We refer to $[1,4]$ for further details.

\section{Optimal Energy Flow Management in Multicarrier Energy Networks}

\subsection{Problem formulation}

Optimal Energy Flow Management in multicarrier energy networks aims at identifying the optimal asset of the control/decision variables $\mathbf{u}$ that minimizes one or more objective functions $f_{i}$ subject to a number of nonlinear

\footnotetext{
${ }^{1}$ This formalism will be more intuitively understood in Section 4, where it will be applied to the peculiar energy hub architecture adopted in this paper;
} 
equality $g_{j}$ and inequality constraints $h_{k}$.

From a mathematical point of view, the overall problem can be formalized by the following constrained nonlinear multiobjective programming problem:

$$
\left\{\begin{array}{cc}
\min _{\mathbf{x}, \mathbf{u}} & f_{i}(\mathbf{x}, \mathbf{u}) \quad i=1, . ., p \\
\text { s.t. } & g_{j}(\mathbf{x}, \mathbf{u})=0 \quad j=1, . ., n \quad(7) \\
& h_{k}(\mathbf{x}, \mathbf{u})<0 \quad k=1, . ., m
\end{array}\right.
$$

Where $\mathbf{x}$ is the vector of dependent variables, $p$ is the number of scalar objective functions, $n$ is the number of equality constraints and $m$ is the number of inequality constraints.

The scalar equations formalized in (7) can be expressed in a more compact vector formalism as follow:

$$
\left\{\begin{array}{cc}
\min _{\mathbf{x}, \mathbf{u}} & \mathbf{f}(\mathbf{x}, \mathbf{u}) \\
\text { s.t. } & \mathbf{g}(\mathbf{x}, \mathbf{u})=0 \\
\mathbf{h}(\mathbf{x}, \mathbf{u})<0
\end{array}\right.
$$

Where $\mathbf{f}(\mathbf{x}, \mathbf{u})$ is the $p$-dimensional objective function vector, $\mathbf{g}(\mathbf{x}, \mathbf{u})$ is the $n$-dimensional equality constraint vector and $\mathbf{h}(\mathbf{x}, \mathbf{u})$ is the $m$-dimensional vector describing the inequality constraints.

The control/decision variables include the active power generated by the available electrical generators, the set points of the primary voltage controllers, the pressures at the gas production nodes and the state variables describing the operation points of each energy hub (also known as the dispatch factors).

The dependent variables include the voltage magnitude and phase angle at electrical load buses, the voltage phase angle and the reactive power generated at the electrical generating buses, the active and reactive power generated at the electrical slack bus and the pressures at the gas delivery nodes.

The inequality constraints $\mathbf{h}(\mathbf{x}, \mathbf{u})$ include the maximum allowable power flows for the $n_{L}$ power lines $\left(A_{\text {line }, i}(\mathbf{x}, \mathbf{u}) \leq A_{\text {line }, i}^{\max } \quad i=1, . ., n_{L}\right)$, the maximum allowable gas flow for the $n_{G}$ pipelines $\left(q_{i}(\mathbf{x}, \mathbf{u}) \leq q_{\max , i} \quad i=1, . ., n_{G}\right)$, the minimum and maximum capacity limits for each converter of the $n_{E}$ energy hubs $\quad\left(\mathbf{P}_{\min , i} \leq \mathbf{P}_{i}(\mathbf{x}, \mathbf{u}) \leq \mathbf{P}_{\max , i} \quad i=1, . ., n_{E}\right)$, the minimum and maximum allowable limits for each control/decision variable $\quad\left(u_{\min , i} \leq u_{i} \leq u_{\max , i} \quad i=1, . ., n_{u}\right) \quad$ and $\quad$ for $\quad$ each dependent variable $\left(x_{\min , i} \leq x_{i} \leq x_{\max , i} \quad i=1, . ., n_{x}\right)$.

In addition, the control/decision and the dependant variables should satisfy the power flow equations, the natural gas flow equations and the energy hub equations which represent the equality constraints for problems (7),(8). The objective functions $\mathbf{f}(\mathbf{x}, \mathbf{u})$ could integrate both technical and economic criteria including the minimization of the hourly energy costs, the minimization of the energy losses, the minimization of the voltage deviations etc. Because these design objectives are competing, the optimal energy flow problem has no unique solution and a suitable trade-off between the objectives needs to be identified.

\subsection{The proposed solution paradigm}

The optimal energy flow problem formalized in (7)-(8) involves more than one objective function to be simultaneously optimized. To address this problem proper optimality criteria should be defined. To this aim many solution techniques refer to the concept of Pareto optimality which aims at identifying proper trade-offs 
between the problem objectives [14]. In details, a solution is called Pareto optimal if none of the objective functions can be improved in value without degrading some of the other objective values. Without an additional external preference criteria, all Pareto optimal solutions could be considered equally acceptable by the analyst. Consequently solving the optimal energy flow problem asks for computing all or a representative set of Pareto optimal solutions. Anyway, these solutions cannot be computed efficiently in many cases since they are often of exponential size and NP-hard to compute. As a consequence, approximation methods are frequently used.

The simpler algorithm aimed at approximating the domain of the Pareto optimal solutions is the so called weighting strategy (a.k.a. scalarization method) [14]. This approach aims at combining the multiple problem objectives into one single-objective scalar function. To this aim a positively weighted convex sum of the objectives is typically adopted. By varying these weights it is possible to obtained Pareto optimal solutions but only for problems characterized by convex Pareto fronts.

The main limitation deriving by the application of this solution paradigm in solving the optimal energy flow problem is that the weighting coefficients of the objective function do not necessarily allow trade-offs between the objectives to be expressed especially when the objectives are competing and the number of objectives increases. Further on the weighting strategies suffer of the so-called convexity problem that, in some cases, does not allow the analyst to explore the whole solutions space.

In order to fix this issue, a two stage solution paradigm based on the goal attainment method [11] is here conceptualized. The proposed paradigm allows the analyst to take into account the multi-criteria aspect of the optimal energy flow management problem by defining a set of design goals associated with the set of design objectives, together with a set of under or over attainment factors. The insight principle is to first compute the utopia points of each objective function by solving the following scalar optimization problems:

$$
\left\{\begin{array}{cc}
\min _{\mathbf{x}, \mathbf{u}} & f_{i}(\mathbf{x}, \mathbf{u}) \\
\text { s.t. } & \mathbf{g}(\mathbf{x}, \mathbf{u})=0 \\
\mathbf{h}(\mathbf{x}, \mathbf{u})<0 & i=1, . ., p
\end{array}\right.
$$

The obtained solutions are then assumed as design goals of the optimal energy flow problem and further processed by a goal attainment programming algorithm. According to this approach the final trade off solution is obtained by solving the following scalar constrained optimization problem [12]:

$$
\begin{cases}\min _{\gamma \in \Re} \alpha & \left\{\begin{array}{l}
f_{1}(\mathbf{x}, \mathbf{u})-\gamma \omega_{f 1} \leq f_{1}^{*} \\
\cdots \\
f_{p}(\mathbf{x}, \mathbf{u})-\gamma \omega_{f p} \leq f_{p} *
\end{array}\right. \\
\text { s.t. } \quad & \mathbf{g}(\mathbf{x}, \mathbf{u})=0 \\
& \mathbf{h}(\mathbf{x}, \mathbf{u})<0\end{cases}
$$

where $\gamma$ is the scalar to minimize, $f_{i}^{*}$ is the $i-t h$ design goal while $\omega_{f i}$ is the degrees of under or over achievement of the $i-t h$ goal. The latter can be fixed by the analyst in order to implement proper weighting strategies aimed at indicating the relative importance of each objective function.

The main feature of this programming algorithm is that it is not subject to any kind of convexity limitations [12]. Besides it has been shown to be an effective strategy to solve complex engineering problems characterized by non linear, multimode, vector objective functions. 


\section{Case study}

This section discusses the application of the proposed solution paradigm in the task of solving the optimal energy flow problem for the 30 IEEE test system schematically depicted in fig.1.

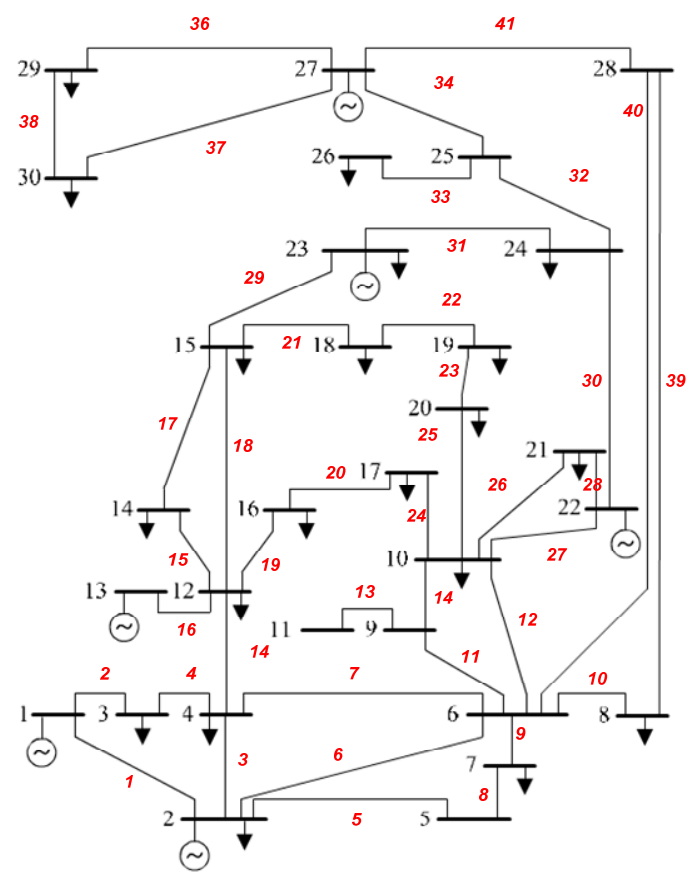

Figure 1: The analyzed multicarrier energy network. The energy hub interconnections are denoted by using red fonts.

The analyzed multicarrier energy network is composed by two overlapped infrastructures, namely the electrical power system and the natural gas network. It integrates 6 sourcing buses, 41 power lines, 41 gas pipelines and 24 energy hubs. We assumed the same topology for both the electrical and the gas network. We considered the 6 sourcing buses as the external interfaces of the multicarrier energy network with the electrical and natural gas markets.

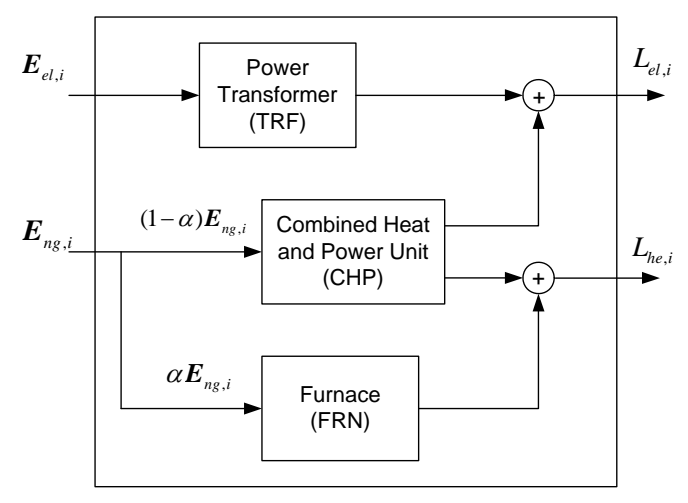

Figure 2: The energy hub architecture

The energy hub architecture considered in our study is depicted in fig. 2. It integrates a power transformer (TRF), a combined heat and power unit (CHP) and a gas furnace (FRN). The coupling matrix describing the $i-t h$ energy hub is defined as follow: 


$$
\left[\begin{array}{c}
L_{e l, i} \\
L_{h e, i}
\end{array}\right]=\left[\begin{array}{cc}
\eta_{e l}^{T R F, i} & \left(1-\alpha_{i}\right) \eta_{e l}^{C H P, i} \\
0 & \left(1-\alpha_{i}\right) \eta_{t h}^{C H P, i}+\alpha_{i} \eta_{t h}^{F R N, i}
\end{array}\right]\left[\begin{array}{c}
E_{e l, i} \\
E_{n g, i}
\end{array}\right]
$$

Where $\alpha_{i}$ is the $i$-th dispatch factor describing the allocation of the natural gas energy flow $E_{n g, i}$ between the CHP and the FRN.

We assumed as control variables the active electrical energy and the natural gas acquired during the fixed control period $\Delta t$ by the 6 sourcing buses namely:

$$
\left.\mathbf{u}=\left[P_{1}^{S P} \Delta t, P_{2}^{S P} \Delta t, P_{5}^{S P} \Delta t, P_{8}^{S P} \Delta t, P_{11}^{S P} \Delta t, P_{13}^{S P} \Delta t, q_{1} \Delta t, q_{2} \Delta t, q_{5} \Delta t, q_{8} \Delta t, q_{11} \Delta t, q_{13} \Delta t\right]\right)(1
$$

While the dependent variables are represented by the pressure at all natural gas buses, the voltage magnitude at all electrical buses, the voltage phase angle at all electrical buses except the slack bus and the energy hub dispatching factors namely:

$$
\mathbf{x}=\left[V_{1}, . ., V_{30}, \theta_{2}, . ., \theta_{30}, p_{1}, . ., p_{30}, \alpha_{1}, . ., \alpha_{24}\right](13)
$$

Three objectives have been considered in our study namely:

- the minimization of the energy costs computed as:

$$
\begin{aligned}
& f_{1}=\left(P_{1}^{S P} \Delta t+P_{2}^{S P} \Delta t+P_{5}^{S P} \Delta t+P_{8}^{S P} \Delta t+P_{11}^{S P} \Delta t+P_{13}^{S P} \Delta t\right) c_{E}(t)+ \\
& +\left(q_{1} \Delta t+q_{2} \Delta t+q_{5} \Delta t+q_{8} \Delta t+q_{11} \Delta t+q_{13} \Delta t\right) c_{N G}(t)
\end{aligned}
$$

where $c_{E}(t)$ and $c_{N G}(t)$ are the unit cost of the active electrical energy and the natural gas during the fixed control period respectively;

- the minimization of the active electrical losses computed as:

$$
f_{2}=\left(\sum_{i \in n G} P_{i}^{S P}-\sum_{j \in n L} P_{j}^{S P}\right) \Delta t
$$

where $n G$ and $n L$ indicate the list of the sourcing buses and the load buses respectively;

- the minimization of the natural gas losses ${ }^{2}$ computed as:

$$
f_{3}=\left(\sum_{i \in n G} q_{i}-\sum_{j \in n L} q_{j}\right) \Delta t
$$

As far as the equality constraints are concerned, we considered the power flow equations describing the active power injected on all electrical buses and the reactive power injected at the electrical load buses for the considered control time namely:

$$
\begin{aligned}
& P_{i}^{S P}=V_{i} \sum_{j=1}^{N} V_{j} Y_{i j} \cos \left(\delta_{i}-\delta_{j}-\theta_{i j}\right) \quad i \in[1, . .30] \\
& Q_{j}^{S P}=V_{j} \sum_{k=1}^{N} V_{k} Y_{j k} \sin \left(\delta_{j}-\delta_{k}-\theta_{j k}\right) \quad j \in n L
\end{aligned}
$$

And the energy hub balancing equations:

$$
\left[\begin{array}{c}
L_{e l, i} \\
L_{h e, i}
\end{array}\right]=\left[\begin{array}{cc}
\eta_{e l}^{T R F, i} & \eta_{e l}^{C H P, i} \\
0 & \left(1-\alpha_{i}\right) \eta_{t h}^{C H P, i}+\alpha_{i} \eta_{t h}^{F R N, i}
\end{array}\right]\left[\begin{array}{c}
E_{e l, i} \\
E_{n g, i}
\end{array}\right] \quad i \in[1,24]
$$

\footnotetext{
${ }^{2}$ In this context it is important to clarify how we compute the "natural gas losses". In details, the proposed model does not allow us to compute the physical energy loss via pipeline (i.e. it does not consider a loss rate per unit length of pipelines of per unit junction/branching). On the contrary it allows us to estimate the efficiency of the gas transmission network by computing the sum of the power demand of the compressors installed in the network. This quantity has been considered as an objective function of the optimization problem.
} 
Finally we considered 142 inequality constraints describing the maximum allowable apparent power flow on each power line, the maximum allowable gas flow for the gas pipelines and the minimum and maximum allowable values for the voltage magnitude at all electrical buses namely:

$$
\left\{\begin{array}{l}
A_{\text {line }, i}(\mathbf{x}, \mathbf{u}) \leq A_{\text {line }, i}^{\max }=1 \quad i \in[1,41] \\
q_{i}(\mathbf{x}, \mathbf{u}) \leq q_{\max , i}=1 \quad i \in[1,41] \\
0.9 \leq V_{i} \leq 1.1 \quad i \in[1,30]
\end{array}\right.
$$

The active electrical energy $L_{e l, i}$ and thermal energy $L_{h e, i}$ supplied by the energy hubs network are depicted in fig. 3a and $3 \mathrm{~b}$ respectively ${ }^{3}$. We assumed a control period of one hour and an hourly cost of $38.45 € / \mathrm{MWh}$ and $18 € / \mathrm{MWh}$ for the active electrical energy and the natural gas respectively.

Starting from these data we solved the optimal energy flow problem formalized in (10) by applying the proposed computing paradigm. To ensure the same percentage of under- or overattainment of the design objectives we assumed $\omega_{f i}=\left|f_{i} *\right|$. The obtained results are summarized in fig. 4 where the state vector describing the actual multicarrier energy network operation is depicted.

Analyzing these profiles it is worth nothing as the corresponding state variables vector satisfies both the equality and the inequality constraints describing the secure and reliable operation of both the electrical and the natural gas network. Besides it allows the analyst to effectively deploy multiple operating criteria by exploiting the couplings and the interactions between the two energy infrastructures.

This is also confirmed by analyzing tab. I where the utopia points, the obtained objective function values and the corresponding percent deviations for the three design objectives are reported. Analyzing these results it is worth observing as the proposed methodology converges to a solution that is very close to the utopia points (which are in general unattainable). Such a solution is usually referred as a compromise solution and it satisfies the Pareto optimality conditions, namely [13].

These important features allow the analyst to optimize systematically and simultaneously a collection of objective functions describing both technical and economic criteria as far as to satisfy the large set of heterogeneous constraints aimed at ensuring the safe operation of the multicarrier energy network.

These benefits have been confirmed by further studies aimed at solving the optimal energy flow problem in the presence of time varying cost and load patterns. In details we considered a 12 hour time scenario with a 1 hour control period. The hourly profiles of the energy costs and the electrical $\left(L_{e l, i}\right)$ and the thermal $\left(L_{h e, i}\right)$ load supplied by each energy hub are reported in figs. 5 and 6 respectively ${ }^{4}$.

The obtained results have been summarized in figs. 7-9. In details fig.7 reports the hourly evolution of the dependent variables for both the electrical and the natural gas network. The corresponding profiles of the energy flows demanded by the energy hubs network are depicted in fig. 8. Analyzing these figures it is worth observing as the proposed methodology converges to a compromise solution which satisfy both the equality and the inequality constraints for all the multicarrier network operation states. This feature allows an effective and reliable multicarrier network operation also in the presence of variable load and energy cost volatilities.

This is also confirmed by the results reported in fig. 9, where the hourly evolution of the utopia points and the actual values of the objective functions are depicted. In the same figure the time profiles of the solutions

\footnotetext{
${ }^{3}$ In this context it is important to clarify that the patterns of active electrical energy and heat demands reported in fig. 3 have been defined according to the profiles reported in [4]. This is only a work assumption that does not affect the validity of the proposed methodology.

${ }^{4}$ In fig. 6 and subsequent ones, each color represents a hourly profile for a particular energy hub.
} 
computed by solving the optimal energy flow problem by a traditional scalarization technique are also reported. The latter is based on the solution of the following constrained scalar optimization problem:

$$
\begin{cases}\min _{\mathbf{x}, \mathbf{u}} \sum_{i=1}^{3} \sqrt{\left(\frac{f_{i}(\mathbf{x}, \mathbf{u})-f_{i}^{*}}{f_{i}^{*}}\right)^{2}} \\ \text { s.t. } & \mathbf{g}(\mathbf{x}, \mathbf{u})=0 \\ & \mathbf{h}(\mathbf{x}, \mathbf{u})<0\end{cases}
$$

By analyzing this figure it is worth noting the effectiveness of the goal attainment based programming algorithm in identifying valuable trade offs between the competing design objectives. This is mainly due to a more accurate exploration of the solution space and a more effective aggregation of the objective functions characterizing the goal programming approach ${ }^{5}$.

Finally, it is important to note that the application of the proposed solution paradigm for large scale multicarrier energy systems could require high computational burden. In addressing this flaw, it is possible to exploit the intrinsic parallelism characterizing the proposed algorithm. In this connection the authors developed and tested a powerful parallelization algorithm aimed at effectively deploying the proposed architecture on a distributed computing environment. Due to space limitation, the theoretical background and the obtained experimental results will be presented in a separate paper.

Table I: Design goals vs. actual objective function values

\begin{tabular}{|l|l|l|l|}
\hline & $f_{1}[€ / \mathrm{MWh}]$ & $f_{2}$ [p.u.] & $f_{3}[p . u]$. \\
\hline Utopia Points & 21.895 & 0.0004 & 0.0021 \\
\hline Actual Solution & 23.545 & 0.0007 & 0.0034 \\
\hline $\begin{array}{l}\text { Percentage } \\
\text { deviations }\end{array}$ & 7.5 & 75 & 61 \\
\hline
\end{tabular}

\footnotetext{
${ }^{5}$ In particular, by comparing figs. 5 and 8, it could be noted that although the ration between electricity and natural gas unit prices is lower in hours from 1 to 6 and higher in hours from 7 to 12, the optimal solution indicates that the energy hubs require larger amounts of gas and small amounts of electricity during "off peak hours" from 2 to 5 . This strategy could appear not rational since the CHP unit would be more conveniently operated at high load levels (consuming large amounts of gas) during peak hours from 7 to 12 . This issue could be justified by observing that the algorithm, according to the hypothesis assumed in the case study, is not able to manage the thermal energy generated in excess to the actual heat demand (i.e. no thermal storage is presented in the considered hub architecture). As a consequence it tends to use the CHPs at the highest load levels suitable with the equality constraints describing the heat demand at each energy hub. The important role of the energy storage devices in multicarrier energy networks are currently under investigation by the authors.
} 


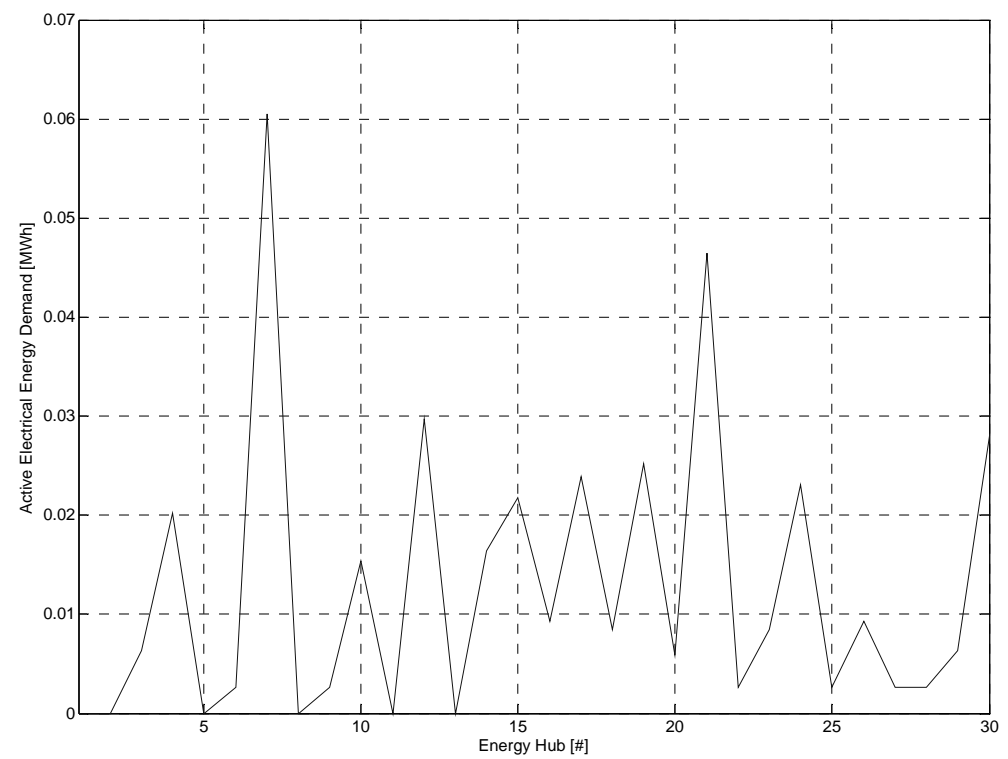

a)

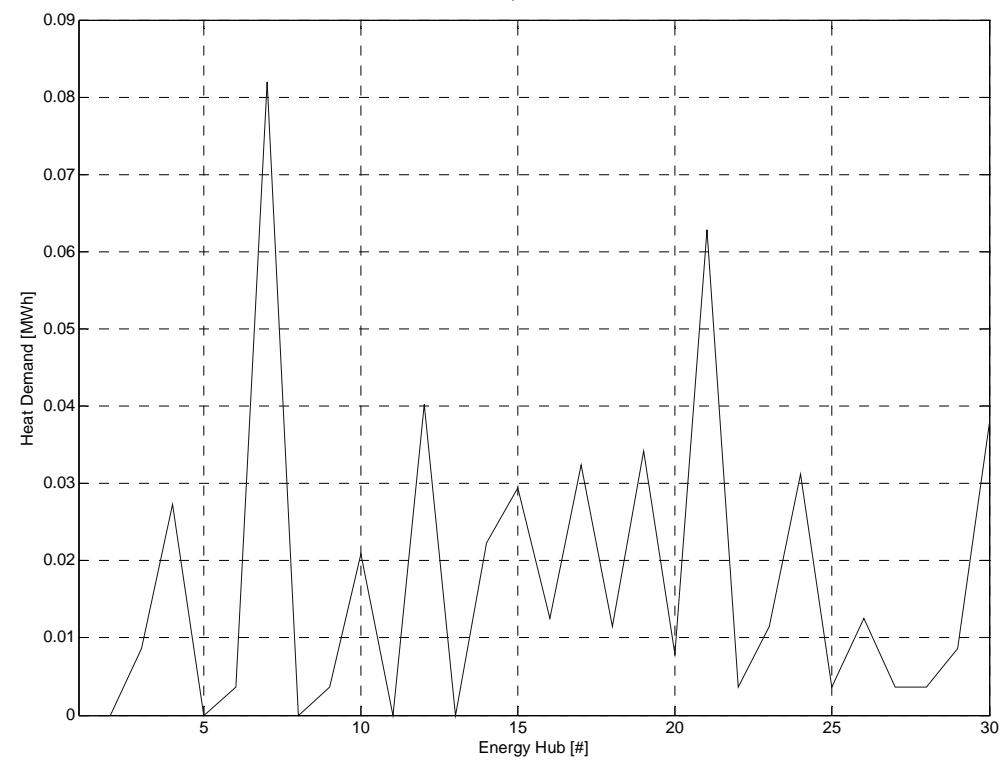

b)

Figure 3: The active electrical energy $L_{e l, i}$ (a) and heat demands $L_{h e, i}(b)$ for the energy hubs network

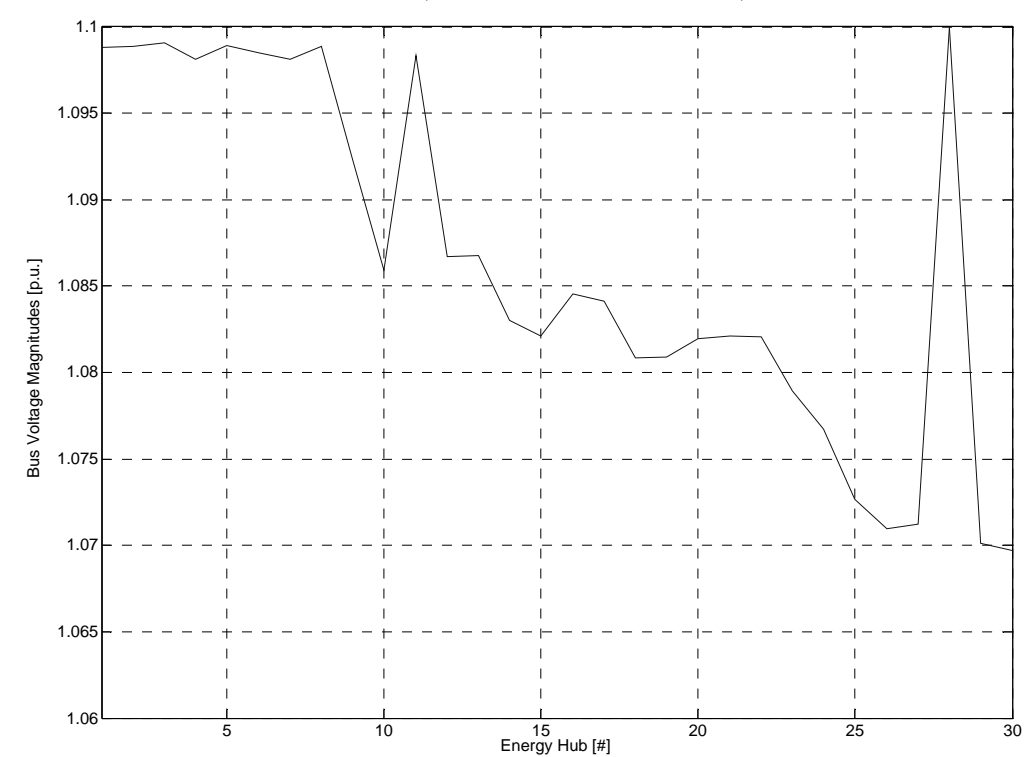




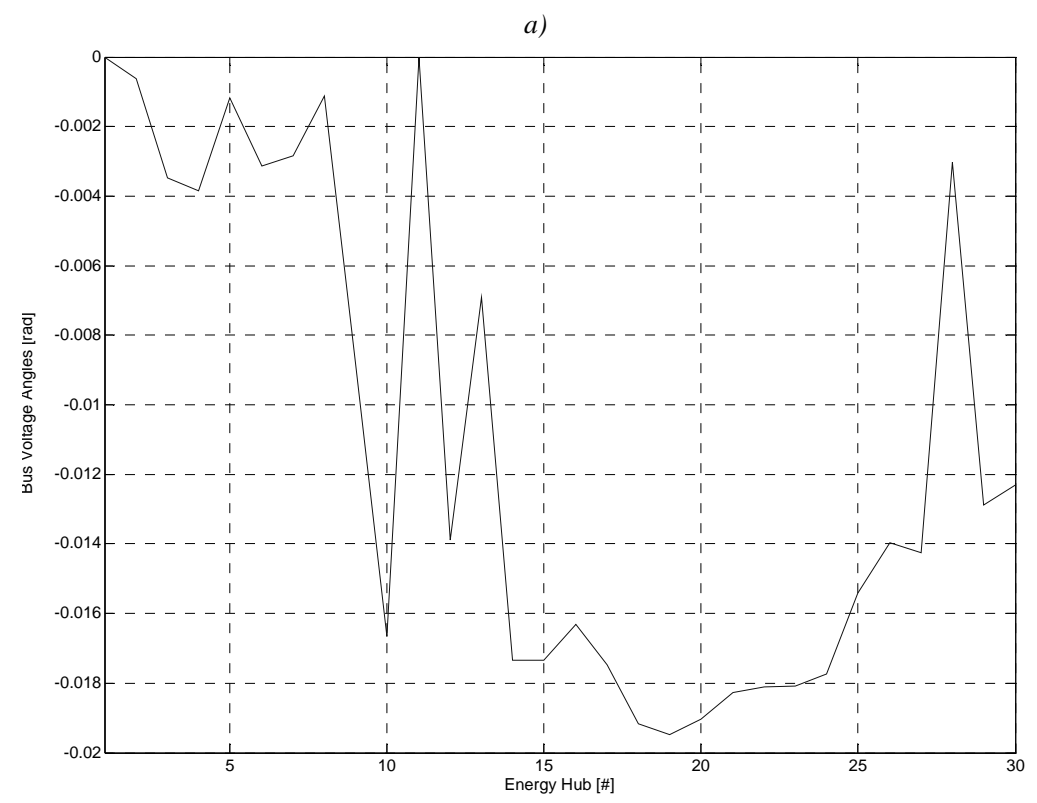

b)

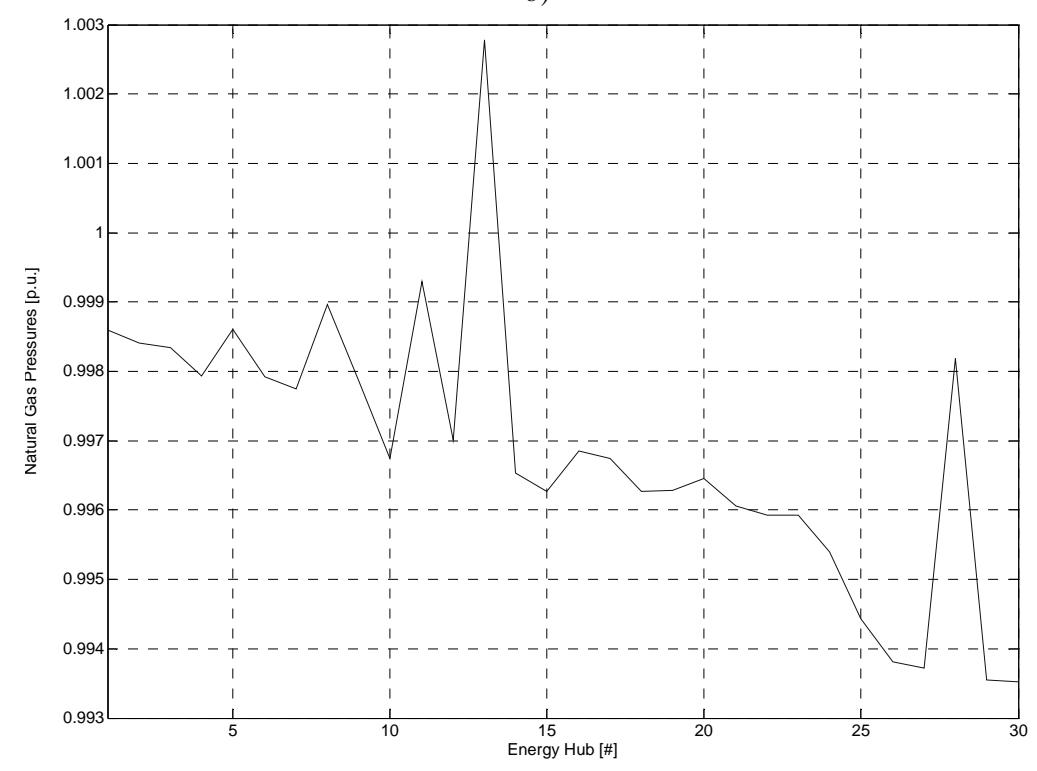

c)

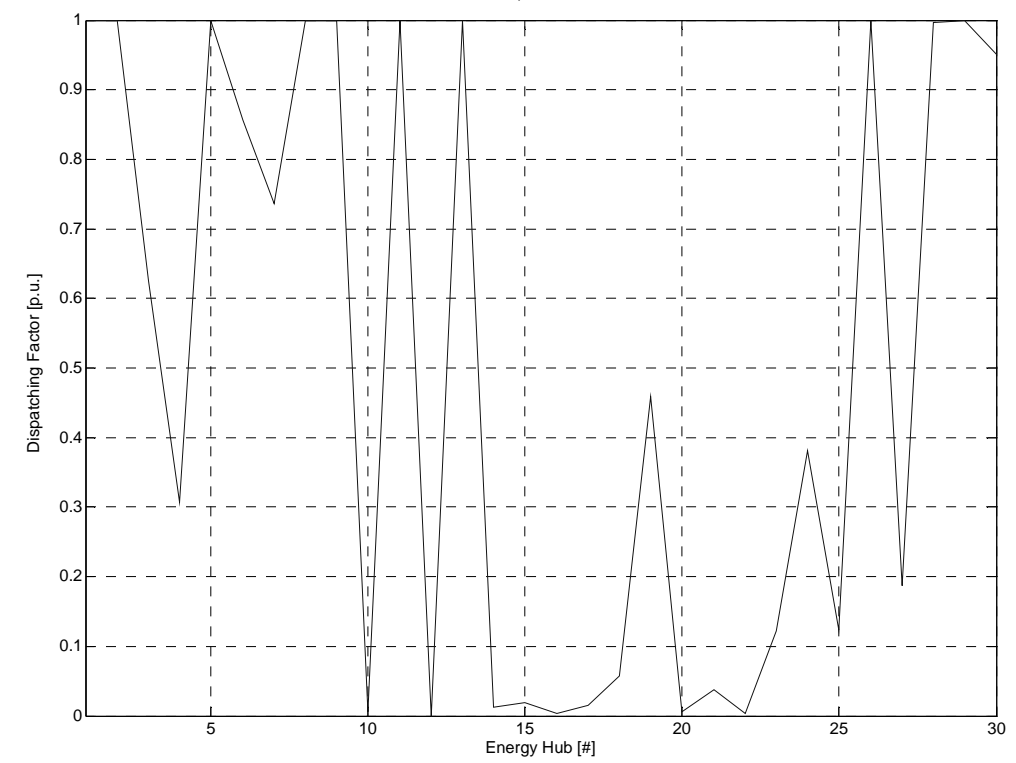


d)

Figure 4: Optimal Energy Flow problem solution computed by the proposed paradigm:

a) Electrical buses voltage magnitudes

b) Electrical buses voltage angles

c) Natural gas buses pressures

d) Energy hub dispatching factors

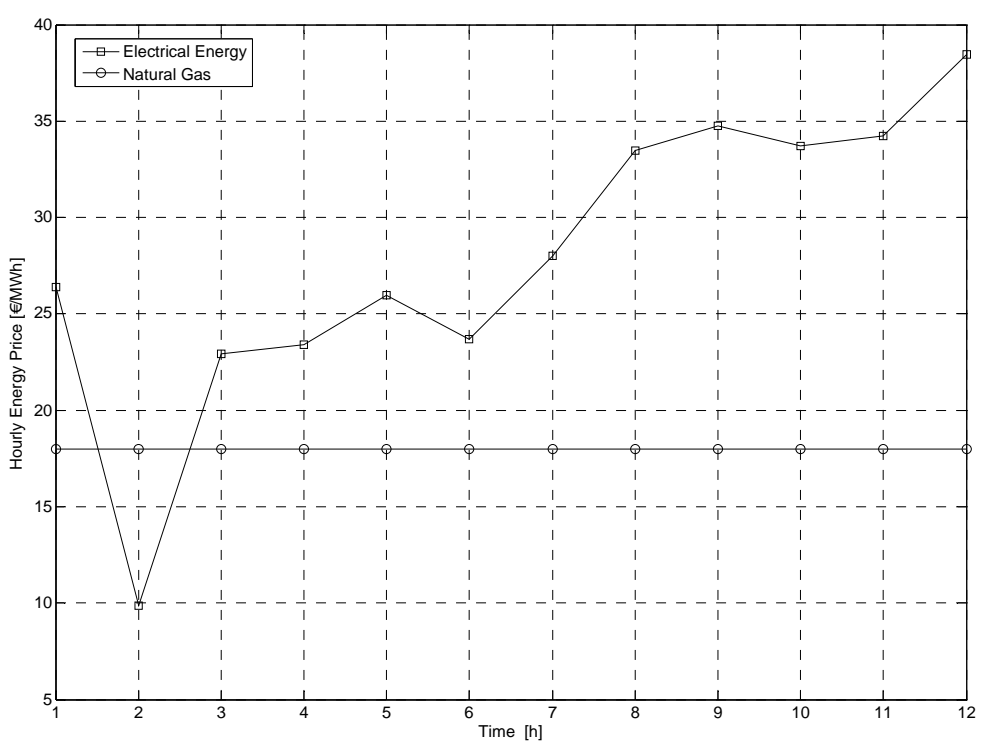

Figure 5: The hourly prices of the electrical energy and the natural gas

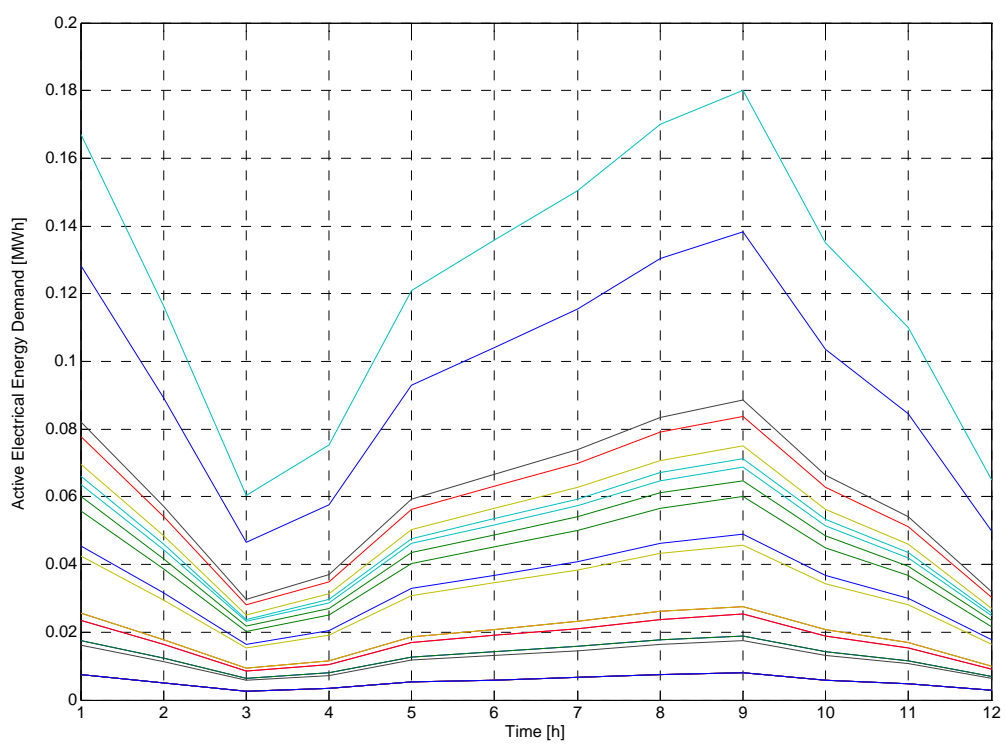

a) 


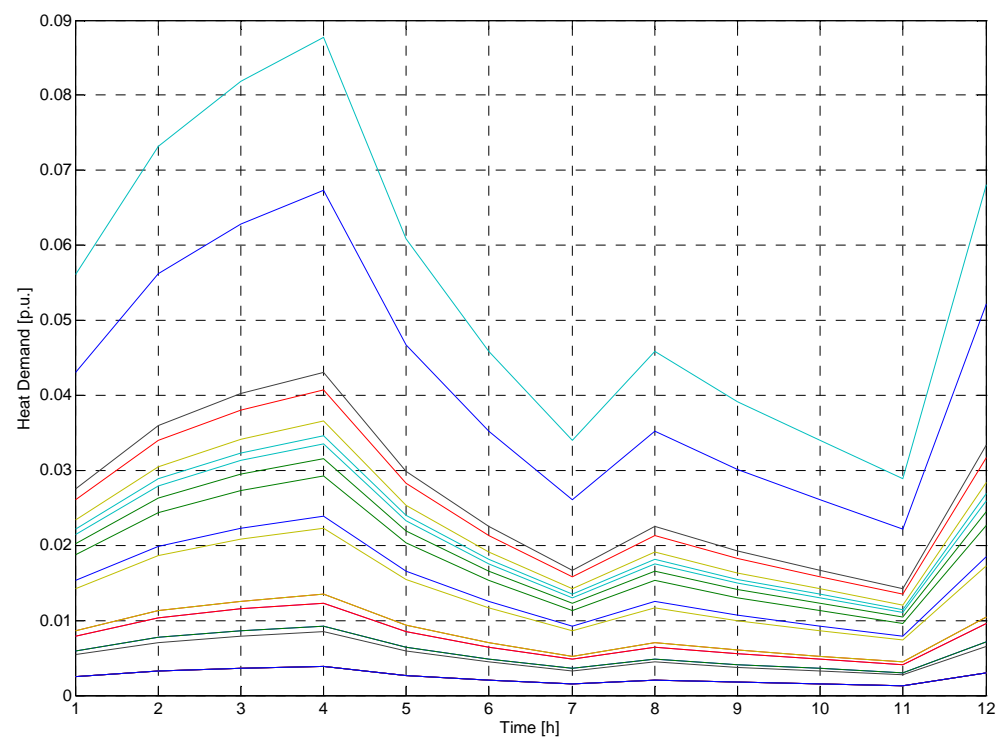

b)

Figure 6: a) Hourly profiles of the electric and (b) thermal load supplied by each energy hub

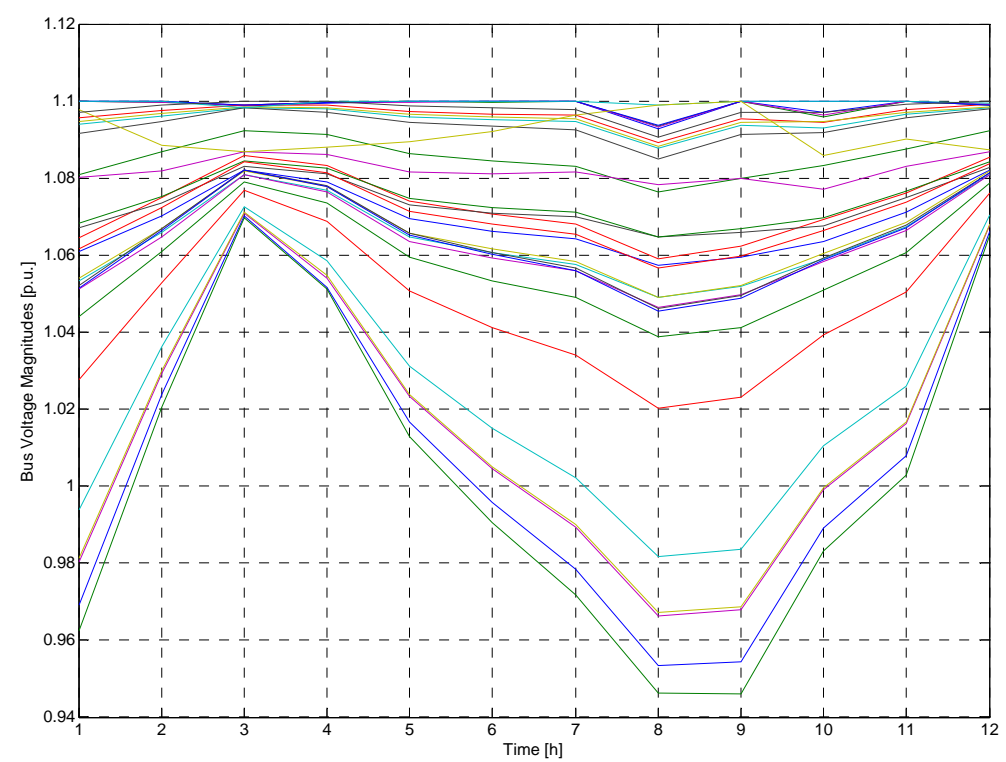

a) 


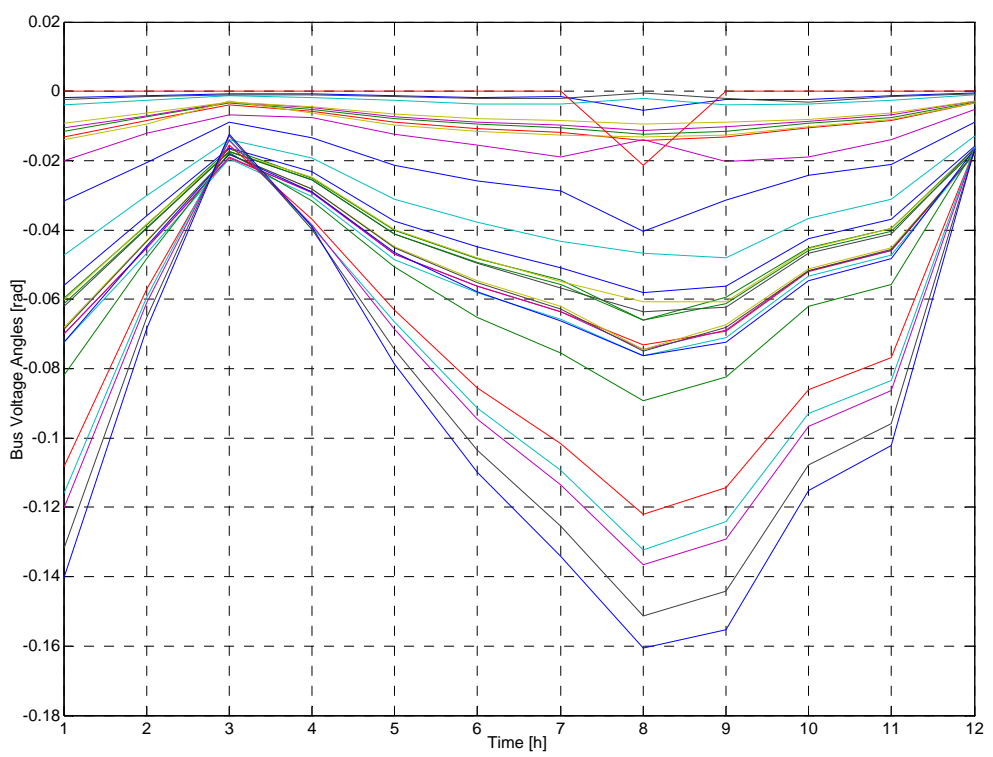

b)

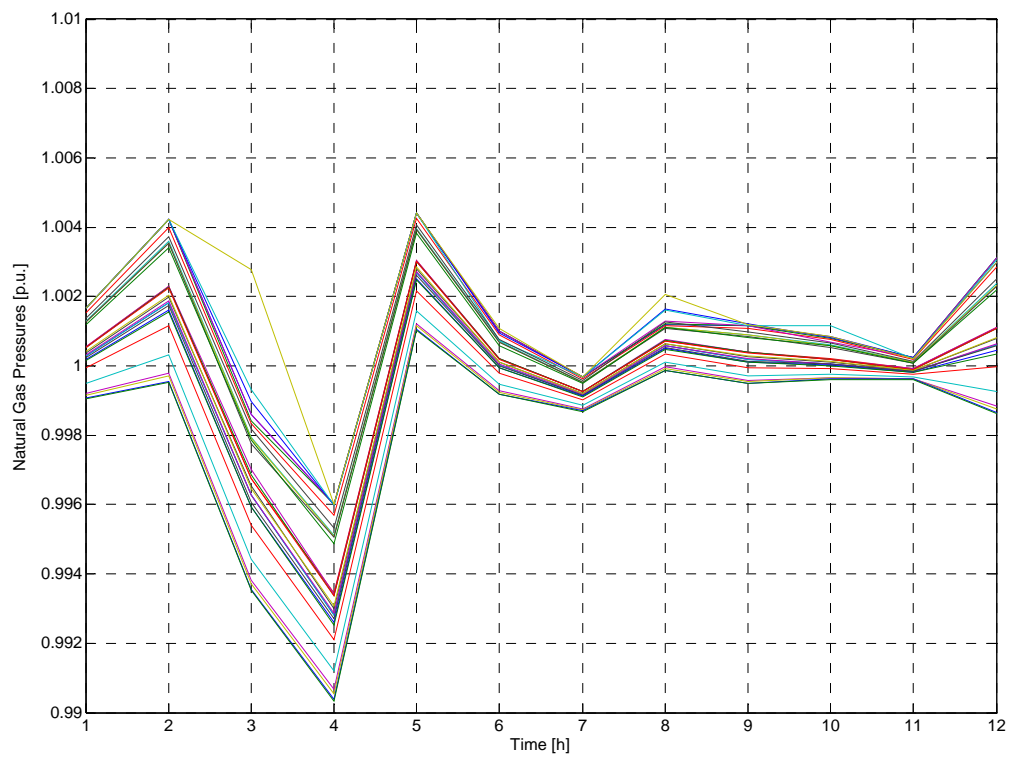

c)

Figure 7: Hourly profiles of the Optimal Energy Flow problem solutions computed by the proposed paradigm: a) Electrical buses voltage magnitudes

b) Electrical buses voltage angles

c) Natural gas buses pressures 


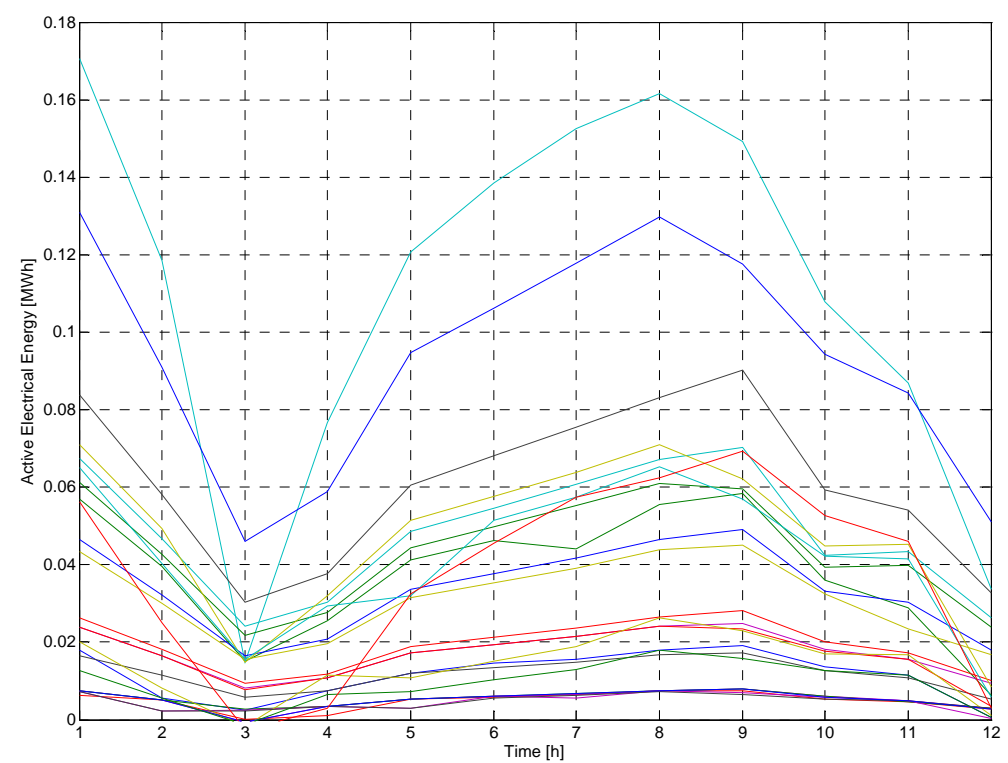

a)

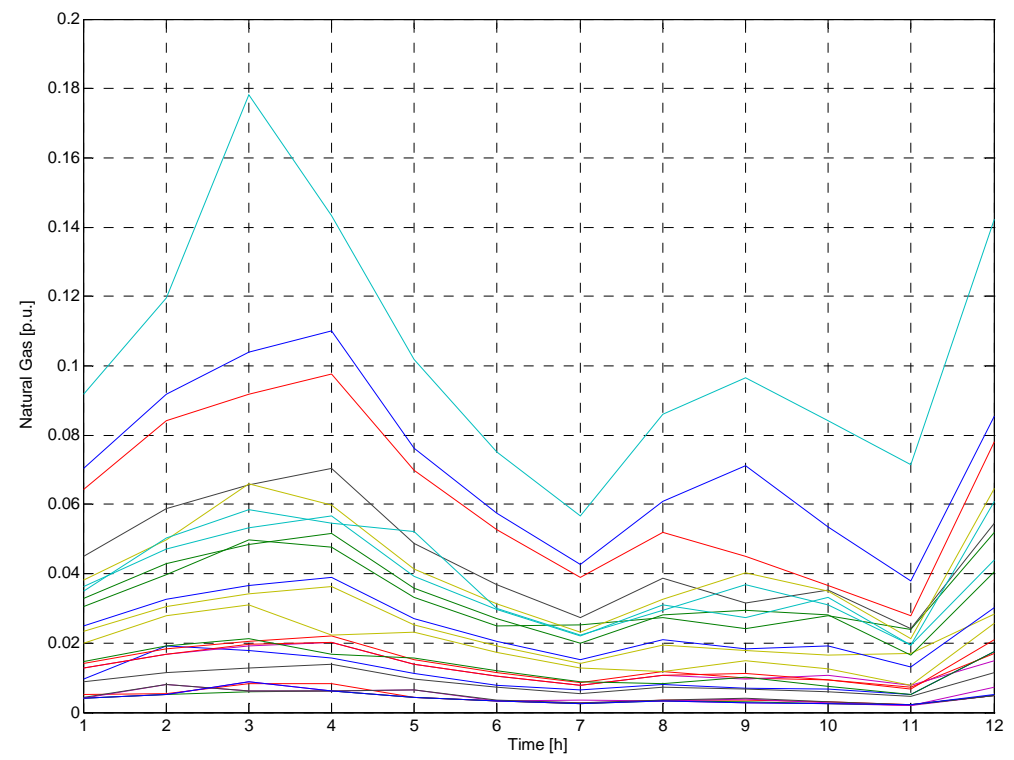

b)

Figure 8: Hourly profiles of the energy flows demanded by the energy hubs network a) Active electrical energy

b) Natural gas 


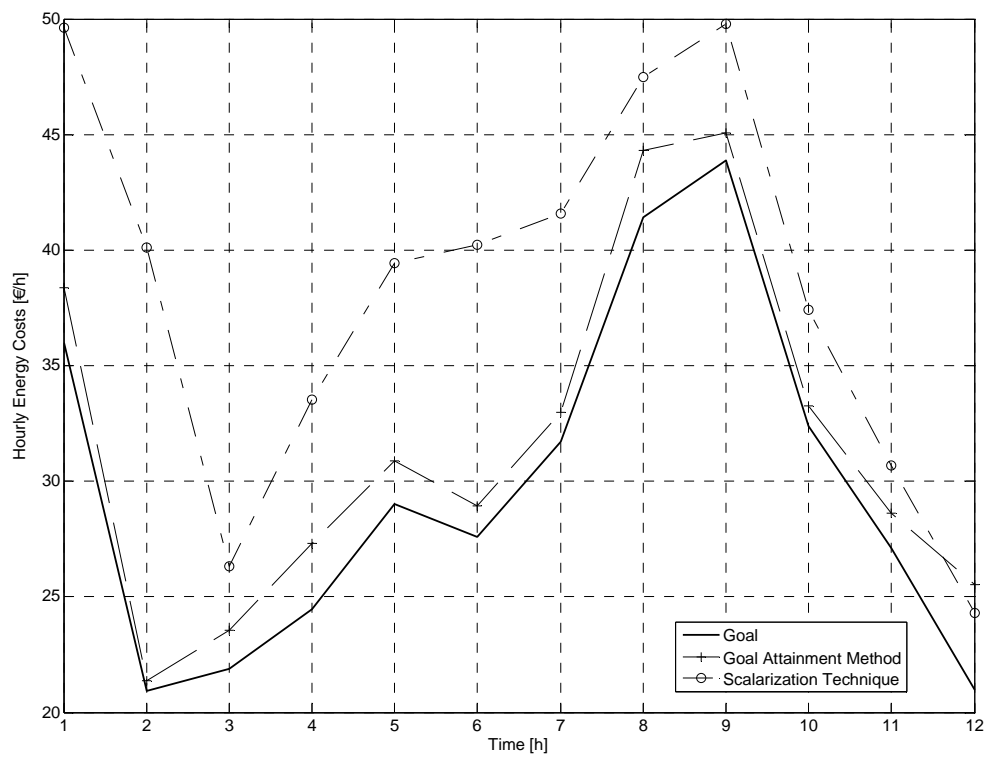

a)

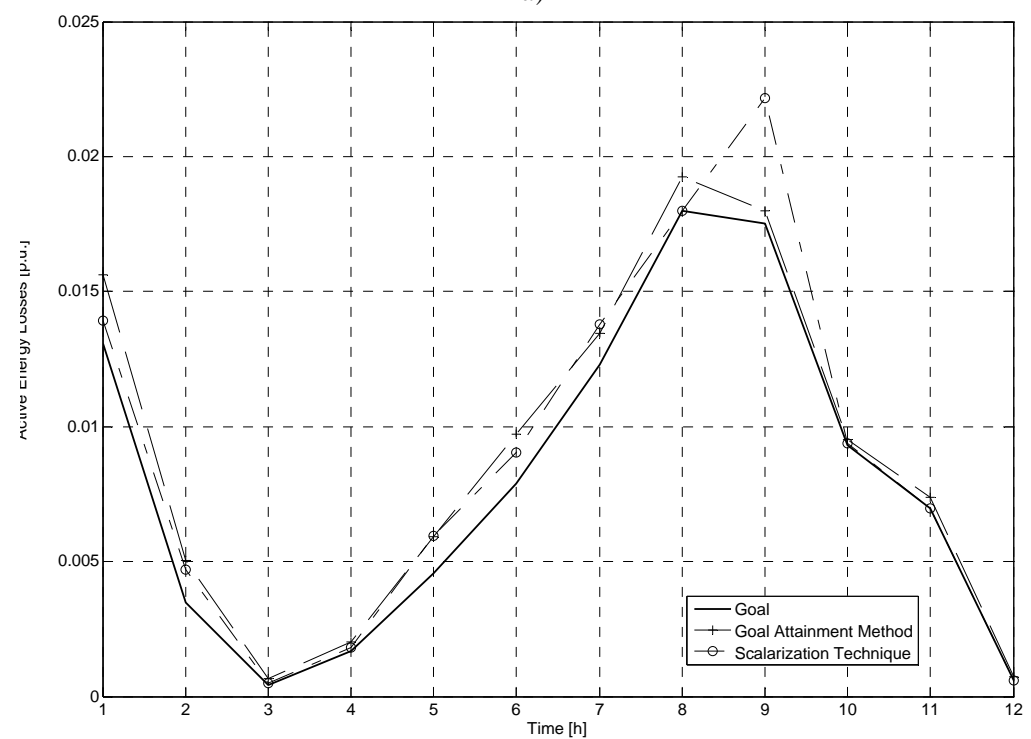

b)

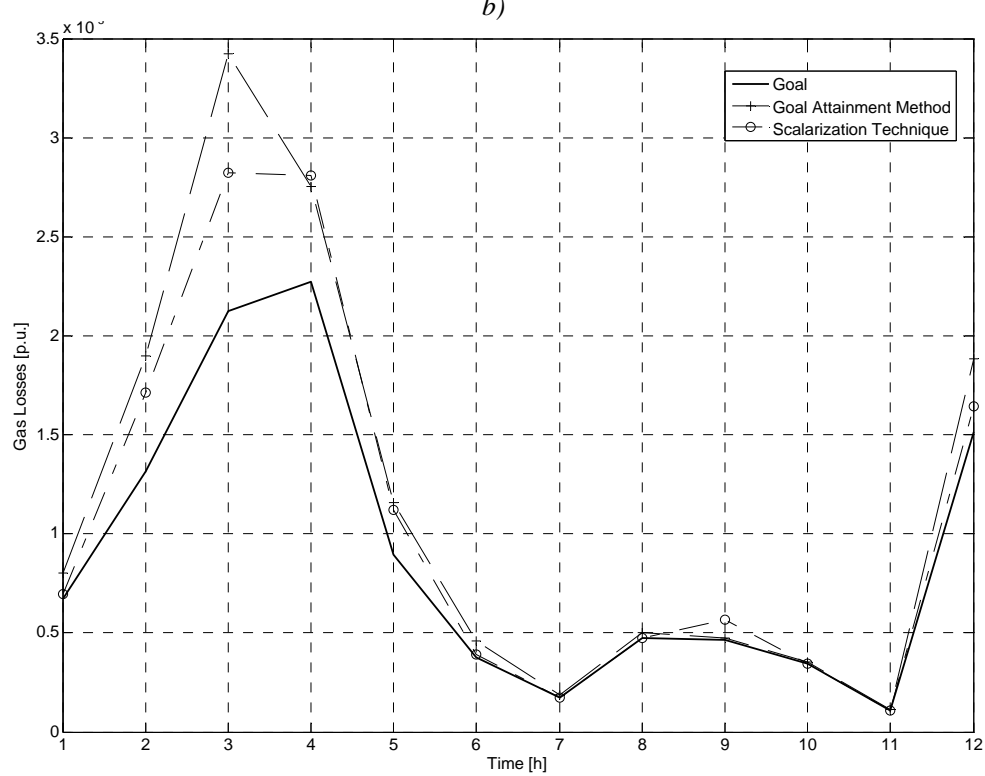

c) 
Figure 9: Comparison of the hourly profiles of the design goals (utopia points) and the actual solution obtained by applying the proposed methodology and a scalarization technique: a) energy costs

b) active electrical energy losses

c) natural gas losses

\section{Conclusions}

In this paper we addressed the problem of optimal energy flow in multicarrier energy networks in the presence of interconnected and distributed energy hubs.

We demonstrated as this problem can be solved by identifying the optimal asset of the energy hubs network which minimize multiple cost functions (descriptive of economic, technical and environmental issues) under equality and inequality constraints. In trying and addressing this issue in this paper we conceptualized a multiobjective optimization methodology based on the goal attainment method. The proposed paradigm allows the analyst to take into account the multi-criteria aspect of the optimal energy flow management problem by defining a set of design goals associated with the set of design objectives, together with a set of under or over attainment factors.

The adoption of this solution approach allowed us to effectively address the optimal energy flow problem also for critical network operating states characterized by high loads and energy prices volatilities. Moreover it allows the energy hubs to provide valuable benefits to the electrical grids, such as alleviating transmission and distribution constraints, providing demand response capability and voltage support and helping to reduce steep peak price curves in the wholesale energy markets. Finally, its intrinsic flexibility allows the analyst to easily integrate further design objectives as far as voltage deviations, regulating costs, power quality indexes are concerned.

Further researches aimed at assessing the validity and the benefits deriving by the application of the proposed methodology on large scale energy systems are currently being conducted by the authors.

\section{References}

[1] Martin Geidl and Göran Andersson, Optimal Power Flow of Multiple Energy Carriers, IEEE Transactions on Power Systems, 22/1 (2007), 145-155.

[2] T. Krause, G. Andersson, K. Fröhlich, A. Vaccaro, Multiple-Energy Carriers: Modeling of Production, Delivery, and Consumption, IEEE Proceedings, 99/1(2011), 15 - 27

[3] F. Kienzle, P. Ahcin, G. Andersson, Valuing Investments in Multi-Energy Conversion, Storage, and Demand-Side Management Systems under Uncertainty, IEEE Transactions on Sustainable Energy, 2/2, (2011), $194-202$.

[4] A. Parisio, C. del Vecchio, A. Vaccaro, A Robust Optimization Approach to Energy Hub Management, International Journal of Electrical Power and Energy Systems, Elsevier, 42/1 (2012), 98-104.

[5] A.Vaccaro, M. Popov, D.Villacci, V. Terzija, An Integrated Framework for Microgrids Modeling, Control, Communication and Verification, IEEE Proceedings, 99/1 (2011), 119-132

[6] G. Bontempi, A.Vaccaro, D.Villacci, Semi-physical modelling architecture for dynamic assessment of power components loading capability, IEE Proceedings - Generation, Transmission and Distribution, 151/4 (2004), 533-542. 
[7] V.Madani, R.L.King, Strategies to meet grid challenges for safety and reliability, International Journal of Reliability and Safety, 2/1 (2008)

[8] A.Vaccaro, C. Canizares, D.Villacci, An Affine Arithmetic based Methodology for Reliable Power Flow Analysis in the presence of Data Uncertainty, IEEE Trans. On Power Systems, 25/2 (2010), 624 - 632

[9] A. Tomasgard, F. Rømo, M. Fodstad, K. Midthun, Optimization models for the natural gas value chain, in G. Hasle, K.-A. Lie, E. Quak (Eds.), Geometric modelling, numerical simulation and optimization, Springer, Berlin 2007, 521-558

[10] F. Rømo, A. Tomasgard, L. Hellemo, M. Fodstad, B. Haukelidsæ Eidesen, B. Pedersen, Optimizing the Norwegian Natural Gas Production and Transport, Interfaces, 39/1 (2009), 46-56

[11] J.L. Cohon, Multiple objective programming and planning, Academic Press, New York , 1978.

[12] V.Galdi, A.Vaccaro, D.Villacci, Voltage regulation in MV networks with dispersed generations by a neural based multiobjective methodology, Electric Power Systems Research, 78/5 (May 2008), 785-793

[13] R.T. Marler, J.S. Arora, Survey of multi-objective optimization methods for engineering, Struct Multidisc Optim 26, 369-395 (2004)

[14] F. Torelli, A.Vaccaro, "A Generalized Computing Paradigm based on Artificial Dynamic Models for Mathematical Programming" to be appear on Soft Computing-Springer 